\title{
Viscosupplementation with intra-articular hyaluronic acid for hip disorders. A systematic review and meta-analysis
}

\author{
Eleonora Piccirilli1 \\ Francesco Oliva ${ }^{1}$ \\ Mihaela Aconstantinesei Murè ${ }^{2}$ \\ Asmaa Mahmoud ${ }^{2,4}$ \\ Calogero Foti ${ }^{2}$ \\ Umberto Tarantino ${ }^{1}$ \\ Nicola Maffulli ${ }^{3}$ \\ 1 Department of Orthopaedics and Traumatology, \\ University of Rome "Tor Vergata", Italy \\ 2 Department of Physical and Rehabilitation Medi- \\ cine, School of Medicine, University of Rome \\ "Tor Vergata", Italy \\ 3 Head of Department of Orthopaedics and Trauma- \\ tology, Azienda Ospedaliera San Giovanni di Dio e \\ Ruggi d'Aragona, University of Salerno, Italy; \\ Queen Mary University of London, Barts and the \\ London School of Medicine and Dentistry, Centre \\ for Sports and Exercise Medicine, Mile End Hospi- \\ tal, London, UK \\ 4 Physical medicine, Rheumatology and rehabilitation \\ department, Faculty of Medicine, Ain Shams \\ University, Cairo, Egypt
}

Corresponding author:

Francesco Oliva

Department of Orthopaedics and Traumatology, University of Rome "Tor Vergata", Italy

E-mail: olivafrancesco@hotmail.com

\section{Summary}

Background: Hip joint diseases are common in adult population and their prevalence increases with age. Osteoarthritis, rheumatoid arthritis and femoroacetabular impingement are the most common chronic diseases in the hip joint. Viscosupplementation with exogenous hyaluronic acid (HA) is one of the most widely used conservative treatment aiming to improve synovial fluid properties and to decrease pain. There is no global consensus on the type of HA, method of injection and frequency, or on its efficacy in hip joint. Methods: We selected published data in English in the PubMed and Google Scholar electronic databases up to March 2016 about hyaluronic acid injections in hip disorders.

Results: 26 articles were included following the Preferred Reporting Items for Systematic Reviews and Meta-Analyses (PRISMA) guidelines.
Conclusion: There is a lack of standardization of HA injections for hip conditions. Our results suggest that this is the best conservative therapy before surgery and it can act on pain relief and function however there is no evidence to prove its ability to modify the morphological structure of the pathological hip and the natural history of the disease. There are few data about the use of HA in other hip disorders rather than osteoarthritis. The most relevant evidence seems to show the utility of HA injections in improving synovial inflammation, but only a few studies have been conducted. Level of evidence: I.

KEY WORDS: hyaluronic acid, hip injections, hip disorders, hip diseases.

\section{Introduction}

Hip disorders are responsible for reduced quality of life in terms of pain, loss of mobility and independence, disability, increased use of health care resources and loss of productivity. In the hip, the prevalence of osteoarthritis $(\mathrm{OA})$ increases with age. In the young men are more affected than women, while over the young age of 45 women are the most affected, with severe limitation of the daily activity and quality of life. The success rate of conservative treatment is limited, and it is often necessary to consider surgical procedure like total hip replacement. While OA is the most common hip disease, different disorders can affect the hip joint. Rheumatoid arthritis is associated with significant morbidity, increased mortality and a complex multifactorial pathogenesis ${ }^{1}$, resulting in systemic autoimmune destruction of bone and joints ${ }^{2}$. Inflammatory cells active macrophages that release cytokines with a significantly contribute to inflammation and joint destruction ${ }^{3}$. Femoroacetabular impingement affects the hip and pelvis with abnormal contact between the acetabular rim ("pincer" impingement) or proximal femur ("cam" impingement). It is increasingly recognized as a potential cause of early hip osteoarthritis and labral cartilage pathology ${ }^{4,5}$. In 1934, Karl Meyer and John Palmer ${ }^{6}$ isolated hyaluronic acid $(\mathrm{HA})$ in humans, identifying it as an endogenous polysaccharide in the extra cellular matrix of many mature tissues including synovial fluid. Various pathological processes change the concentration of HA in synovial fluid. In the early 1960s, Balasz and Denlinger ${ }^{7}$ introduced the idea of visco-supplementation with exoge- 
nous HA to improve synovial fluid properties and to treat articular pain. Shortly afterwards, the first studies on animal models and attempts to treat using $\mathrm{HA}$ were published. In 1997, HA received Food and Drug Administration (FDA) approval in humans. Visco-supplementation involves the use of HA solution to supplement or replace the synovial fluid in a pathological joint to alleviate pain and promote the healing of intra-articular injuries ${ }^{8}$. This procedure should improve the physiological environment of an osteoarthritic joint by restoring the protective viscoelasticity of synovial fluid ${ }^{9-12}$, reducing friction and improving mobility ${ }^{13,14}$. Intra-articular hip injections have been attempted for a wide variety of hip disorders, including $\mathrm{OA}$, rheumatoid arthritis ${ }^{15}$, acetabular labral tears, femoro-acetabular impingement ${ }^{16,17}$. The direct injection of hyaluronic acid in the joint space allows to reach a high concentration with low doses, enhancing a longer permanence in the joint that allows a therapeutic response. Hyaluronic acid preparations have a short half-life and the long term effects cannot solely be attributed to the substitution of molecule itself. This can be mediated by a different process, such as the anti-inflammatory and the anti-nociceptive effects, repair of joint rheology, chondroprotection and normalization of endogenous HA synthesis ${ }^{18}$.

\section{Aim and Methods}

The present review and its procedures were organized, conducted and reported following the Preferred Reporting Items for Systematic Reviews and MetaAnalyses (PRISMA) guidelines ${ }^{19}$. We wished to clarify:

a) the efficacy of HA injections in hip joint diseases;

b) the possibility to modify the natural history of hip OA or all other hip diseases;

c) whether there was a global consensus about the type of HA, technique of injection, number and frequency of injections and outcome measures.

\section{Source of studies and search strategy}

We performed a systematic search (up to September 2016) in PubMed and Google Scholar electronic databases of articles assessing the association between $\mathrm{HA}$ injections and hip joint diseases published in English only. The search strategy covered different type of $\mathrm{HA}$ injections in different hip affections. In the search strategy, we used various combinations of the following key words and MesH terms: hyaluronic acid, hyaluronic injections, hip joint, hip osteoarthritis, hip diseases, rheumatoid arthritis, femoral head avascular necrosis, hip dysplasia, femoroacetabular impingement, hip disorders, hip cartilage, conservative treatment. We only considered for inclusion in the present review the published randomized controlled trials that evaluate clinical outcomes after hyaluronic hip injections performed in any manner. Pilot studies, case reports, editorials, technical notes and narrative review articles were excluded.

\section{Study selection and eligibility criteria}

An orthopaedic surgeon and a physiatry resident performed the search and evaluated the articles independently. An experienced researcher in systematic reviews solved cases of doubt. At the beginning of the procedure, each examiner read the abstracts of all the articles, selected the relevant ones according to inclusion and exclusion criteria previously determined, and then compared the results with the other examiner. After four weeks, the same studies were read again to establish the agreement of the researchers on the selection. No disagreement was observed among the investigators.

\section{Data collection}

One reviewer extracted the data from the full text articles to Excel spreadsheet structured tables to analyze the studies in a descriptive way. The second researcher independently double checked the extraction of primary data from all the articles. Doubts and inconsistencies were followed and solved by discussion. The following information was extracted from articles: aim of the study, number of patients, hip joint disease, type of hyaluronic acid used, type of injections, follow-up and clinical outcomes.

\section{Results}

A total of 35 potentially relevant citations were identified. All of them concerned with hip OA, hip osteoarthritis, rheumatoid arthritis or femoroacetabular impingement. No studies were identified about HA and femoral head avascular necrosis or hip dysplasia. After reviewing titles and abstracts, 10 studies were excluded either because they were not randomized control trials, they were unblinded and uncontrolled studies or they did not clearly investigate the association between HA injections and hip joint disease modifications or because they had very small samples of patients. In addition, some of the excluded studies did not report the presence of a control group. A total of 25 articles were eventually included in the present review. All of them used different models and different protocols, making statistical data analyses impossible. The number of cited references, and other data recruited from the 25 included studies are reported in Table I.

\section{Discussion}

About OA, there is no global consensus on the efficacy of HA in the hip joint.

It is still unclear how HA would act in the joint microenvironment during $\mathrm{OA}$. Recently, Lùrati et al.20 studied the effects of HA visco-supplementation on peripheral $T$ cells in knee and hip $O A$ and they showed that HA injection results in a decrease in 
Table I. HA injections in hip joint diseases.

\begin{tabular}{|c|c|c|c|c|c|}
\hline Reference & Year & Authors & Model & & Frequency of injections \\
\hline 20 & 2015 & Lurati et al. & $\begin{array}{l}86 \mathrm{pz} \\
- \\
-\end{array}$ & $\begin{array}{l}800 \text { and } 1200 \mathrm{kDa} \mathrm{HA} \text { in hip and } \\
\text { knee joint }\end{array}$ & 3 weekly inj \\
\hline 21 & 2016 & Dallari et al. & $\begin{array}{l}111 \mathrm{pz} \\
- \\
- \\
-\end{array}$ & $\begin{array}{l}\text { PRP } \\
\text { PRP + HA } \\
\text { HA }\end{array}$ & 3 weekly inj \\
\hline 22 & 2015 & Battaglia et al. & $\begin{array}{l}46 \mathrm{pz} \\
- \\
-\end{array}$ & $\begin{array}{l}\text { hyaluronic acid (Highly Cross- } \\
\text { Linked HA) }\end{array}$ & 2 inj PRP + 2 inj HA \\
\hline 23 & 2009 & Migliore et al. & $\begin{array}{l}42 \mathrm{pz} \\
- \\
-\end{array}$ & $\begin{array}{l}\text { Hyalubrix } \\
\text { Mepivacaine }\end{array}$ & 2 inj ( 1 per month) \\
\hline 24 & 2009 & Richette et al. & $\begin{array}{l}85 \mathrm{pz} \\
- \\
-\end{array}$ & $\begin{array}{l}\text { HA } \\
\text { Placebo }\end{array}$ & Single inj \\
\hline 25 & 2006 & Quistgaard et al & $\begin{array}{l}185 \mathrm{pz} \\
- \\
- \\
-\end{array}$ & $\begin{array}{l}\text { HA } \\
\text { Corticosteroid } \\
\text { Saline }\end{array}$ & $\begin{array}{l}3 \text { inj HA (each } 14 \text { days) } \\
1 \text { inj corticosteroid } \\
3 \text { inj saline }\end{array}$ \\
\hline 26 & 2008 & $\begin{array}{l}\text { Van De Beckerom } \\
\text { et al }\end{array}$ & $\begin{array}{l}120 \mathrm{pz} \\
-\end{array}$ & 3 hyaluronate formulations & $\begin{array}{l}1 \text { inj } \\
2 \text { or } 3 \text { inj if pain relief }\end{array}$ \\
\hline 27 & 2005 & Tikiz et al & $\begin{array}{l}43 \mathrm{pz} \\
- \\
- \\
\end{array}$ & $\begin{array}{l}\text { Low molecular weight } \mathrm{HA} \\
\text { High molecular weight } \mathrm{HA}\end{array}$ & 3 weekly inj \\
\hline 28 & 2016 & Atchia et al & $\begin{array}{l}77 \mathrm{pz} \\
- \\
- \\
- \\
-\end{array}$ & $\begin{array}{l}\text { No inj } \\
\text { Corticosteroid } \\
\text { Non-animal stabilised HA } \\
\text { Saline }\end{array}$ & Single inj \\
\hline 29 & 2003 & Migliore et al. & $28 \mathrm{pz}$ & $\begin{array}{l}\text { hyaluronan preparations with } \\
\text { molecular weight of } 0.5-0.75 \text { or } \\
1.0 \text { million }\end{array}$ & 1 to 3 inj every 15 days \\
\hline 30 & 2006 & Migliore et al & $\begin{array}{l}30 \mathrm{pz} \\
- \\
\end{array}$ & HA hylan G-F 20 & 1,2 or 3 inj \\
\hline 31 & 2010 & Eyigor et al. & $\begin{array}{l}21 \mathrm{pz} \\
-\end{array}$ & $2.5 \mathrm{ml} \mathrm{HA}$ injection & 3 weekly inj \\
\hline 32 & 2008 & Migliore et al. & $\begin{array}{l}1887 \mathrm{pz} \\
- \\
- \\
\end{array}$ & $\begin{array}{l}\text { Low molecular weight HA } \\
\text { High molecular weight } \mathrm{HA}\end{array}$ & $\begin{array}{l}1 \text { inj } \\
\text { inj every } 6 \text { months if pain } \\
\text { relief }\end{array}$ \\
\hline 33 & 2011 & Migliore et al. & $\begin{array}{l}2343 p z \\
- \\
- \\
\end{array}$ & $\begin{array}{l}\text { Low molecular weight HA } \\
\text { High molecular weight } \mathrm{HA}\end{array}$ & 1 or 2 inj every 6 month \\
\hline 34 & 2006 & Conrozier et al. & $56 \mathrm{pz}$ & HA hylan G-F20 & $\begin{array}{l}\text { 3-4 inj if pain was } \\
\text { unchanged or returned to } \\
\text { baseline levels }\end{array}$ \\
\hline 35 & 2008 & Conrozier et al & $\begin{array}{l}34 \mathrm{pz} \\
- \\
\end{array}$ & Non-animal stabilized HA(NASHA) & Single inj \\
\hline 36 & 2002 & Brocq et al. & $\begin{array}{l}22 p z \\
- \\
\end{array}$ & HA hylan G-F 20 & 1 inj or 2 inj (day 30) \\
\hline 37 & 2003 & Vad et al. & $\begin{array}{l}22 \\
- \\
\end{array}$ & $\begin{array}{l}\text { pz } \\
\text { HA hylan G-F } 20\end{array}$ & 3 inj at 2,3 , and 4 weeks \\
\hline 38 & 2007 & Gaston et al. & $\begin{array}{l}13 \mathrm{pz} \\
- \\
\mathrm{HA}\end{array}$ & synthetic high molecular weight & 3 weekly inj \\
\hline
\end{tabular}


Continued from Table I

\begin{tabular}{|c|c|c|c|c|}
\hline 39 & 2012 & Migliore et al. & $\begin{array}{ll}176 \mathrm{pz} & \\
- & \text { sodium hyaluronate (MW 1,500- } \\
- & 2,000 \mathrm{kDa}) \\
\end{array}$ & $\begin{array}{l}\text { single inj every } 6 \text { months. } \\
\text { Up to } 1 \text { inj per } 3 \text { - month } \\
\text { period, in any } 1 \text { year }\end{array}$ \\
\hline 40 & 2015 & Reum Son A et al. & Rats affected by rheumatoid arthtitis & $\begin{array}{l}\text { Single inj at a volume of } \\
100 \mu \mathrm{L} \text { into the articular } \\
\text { knee joint }\end{array}$ \\
\hline 41 & 2011 & Kim KS et al. & Animal model ${ }^{*}$ & $\begin{array}{l}\text { Intra-articular injection of } \\
\text { HA-Tyr hydrogels } \\
\text { encapsulating } \\
\text { dexamethasone (DMT) }\end{array}$ \\
\hline 42 & 2010 & Yasuda $\mathrm{T}$. & $\begin{array}{l}\text { Cartilage specimens from patients who } \\
\text { underwent total knee replacement who were } \\
\text { diagnosed as having RA or OA }\end{array}$ & Histological evaluation \\
\hline 43 & 2009 & Saito $S$ et al. & 4725 patients in the database & $\begin{array}{l}\text { Single inj of HA or } \\
\text { corticosteroids }\end{array}$ \\
\hline 44 & 2014 & Abate $\mathrm{M}$ et al. & $\begin{array}{ll}23 & p z \\
- & 3 \text { bilateral cases }\end{array}$ & $\begin{array}{l}2 \text { inj (time } 0 \text { and after } 40 \\
\text { days) }\end{array}$ \\
\hline
\end{tabular}

*Lack of information

PZ, patients; INJ, injection; PRP, platelet rich plasma; kDa, kilodalton.

proinflammatory $\mathrm{T}$ cells concentrations. In particular, HA can reduce synovial inflammation and restore the rheological properties of synovial fluid. Some studies compared HA injections to different drugs for hip pain relief. Dallari et. al. ${ }^{21}$ compared Platelet Rich Plasma (PRP) injections to hyaluronic acid (HA) or their association (PRP1HA) at 6 and 12 months follow-up evaluating VAS scale, Harris Hip Score and WOMAC scores in hip OA. Intra-articular PRP injections lead to a significant clinical and functional improvement, with no significant differences between PRP and PRP1HA treatment. Battaglia et al. ${ }^{22}$ combined HA and PRP injections following a specific protocol for moderate to severe hip OA (4 injections), and they performed laboratory, VAS and radiological evaluation after $1,3,6$ and 12 months. The results showed that in $93.7 \%$ of cases there was a significant reduction of subjective pain (VAS scores) and consumption of NSAIDs greater than the HA or platelet gel alone.

Migliore et al. ${ }^{23}$ compared HA injections to Mepivacaine at 6 months follow-up (2 injections): patients in the HA group exhibited a significantly reduced Lequesne's algofunctional index 3 and 6 months after treatment with a significantly reduced pain score (VAS) at 3 and 6 months after treatment when compared with the local anesthetic group.

If compared to placebo, it is not clear whether HA is more effective in the treatment of hip OA. Richette et al. ${ }^{24}$ performed a single injection of $\mathrm{HA}$ or placebo. VAS and WOMAC were evaluated after 3 months and compared to baseline. The results showed that there was no significant difference in terms of efficacy after one injection of HA compared with placebo in patients with hip osteoarthritis.

Comparison between three HA, one corticosteroid and three isotonic saline ultrasound-guided injections was performed by Quistgaard et al. ${ }^{25}$ : the study demonstrated that patients treated with corticosteroids experienced significant improvement in "pain on walking" dur- ing the 3 months of intervention. No such results were experienced after HA injections probably because corticosteroids act immediately on pain relief.

Van De Beckerom et al. ${ }^{26}$ compared three different hyaluronate formulations and evaluated functional improvement, pain relief and also the delay in performing a total hip arthroplasty after two injections. They showed that the three HA formulations do not differentiate in terms of clinical and functional outcomes. These data show that it is still unclear how HA products affect OA treatment outcome based on their different molecular weight.

Some Authors compared the efficacy of lower and higher molecular weight visco-supplementation in the treatment of hip OA. Tikiz et al. ${ }^{27}$ performed three hip injections (one injection per week), and evaluated VAS scale, WOMAC and Lequesne index after 1, 3 and 6 months. Both types of visco-supplementation produced a significant clinical improvement during the 6-month follow-up period, with no significant difference between higher and lower molecular weight hyaluronic acid. Atchia et al. ${ }^{28}$ reported no significant improvement in pain and function after a single high molecular weight $\mathrm{HA}$ injection in moderate to severe hip OA when compared to standard care with no injections or to saline or non animal stabilized HA. This suggests that high molecular weight hyaluronic acids may not have a role to play in moderate to severe hip osteoarthritis.

On a methodological point of view, the latest studies emphasize the importance of ultrasound-guided injections in hip joint. Migliore et al. ${ }^{29}$ reported that ultrasound-guided IA injection of HA in the hip is well tolerated, the few adverse events were local and resolved spontaneously in a few days. Ultrasound guidance is safe and cheap. In addition, the same group of Authors observed the effects HA administered through ultrasound (US)-guided intra-articular injection in patients with symptomatic hip osteoarthritis at 2 and 6 months follow-up and they demonstrated that 
an injection or repeated injections of HA could be effective and acceptable with poor risk ${ }^{30}$. On the contrary, radiological guide is more expensive and it implies a high radiation load. Eyigor et al. ${ }^{31}$ investigated intraarticular HA injection (three injections) through a lateral approach under fluoroscopic control, and reported that this type of injection provided significant reduction in pain scores, disability scores and analgesic use in advanced hip OA. HA injections in hip osteoarthritis seems to reduce the consumption of nonsteroidal anti-inflammatory drugs (NSAIDs). Migliore et al. ${ }^{32}$ reported that NSAIDs consumption decreases already after the first hip injection with low and high molecular weight HA showing that hip viscosupplementation may be effective and cost-saving. A few years later, they increased their population, and they reported that the consumption of NSAIDs was reduced of $48.2 \%$ at the third month when compared with baseline values with an increased sparing effect at 12 th and 24th month ${ }^{33}$.

According to clinical outcome after HA injections, different clinical variables have been considered to evaluate the efficacy in hip osteoarthritis. Conrozier et al. ${ }^{34}$ assessed the clinical response of patients presenting with symptomatic hip osteoarthritis to the first intra-articular HA injection at 3 months of follow up (up to four injections according to symptoms). Clinical response was defined as a $50 \%$ reduction from baseline in the Lequesne index one month after treatment. The response rate was $50 \%$ after the first injection and about half of the patients experienced significant pain relief during the 3 months following a single injection and some of the patients who did not respond to the first injection received significant benefit from a second one.

A few years later, Conrozier et al. ${ }^{35}$ determined that the patient self assessment of treatment efficacy (PATE) after one HA injection was correlated with Lequesne index and walking pain decrease after one $\mathrm{HA}$ injection at 6 months of follow-up; PATE at month 1 was also highly predictive of the efficacy of the injection at month 6 .

In the short term, Brocq et al. ${ }^{36}$ investigated the safety and efficacy of one or two intraarticular HA injectionsin patients with symptomatic hip osteoarthritis. Short-term safety was satisfactory, with a self-limited exacerbation of pain during the first few days in three patients but no infections or other side effects. The response rate was $50 \%$ after the first injection but only one third of the entire population had a sustained improvement at 6 months.

Vad et al. ${ }^{37}$ evaluated the outcome after three HA injections at one year follow-up. HA injections are a valid option for mild to moderate $O A$ according to pain relief but they are not useful in severe OA because they are not able to modify the natural history of the disease and they are not able to avoid surgical procedure.

Gaston et al. ${ }^{38}$ tried to investigate whether there was any relation to pre-injection radiographic changes of osteoarthritis after three visco-supplementations with synthetic hyaluronic acid to the hip joint. HA can alle- viate patient symptoms in hip OA with no significant radiographic changes. Migliore et al. ${ }^{39}$ performed HA injections every 6 months with a maximum of one injection per 3-month period in any 1 year in hip osteoarthritis. Visco-supplementation should be considered as conservative treatment to perform before listing patients for total hip replacement.

Concerning other hip joint diseases rather than OA, few studies investigate the role of $\mathrm{HA}$ injections in rheumatoid arthritis (RA).

Reum Son et al. ${ }^{40}$ considered different preparations for rheumatoid arthritis treatment in rats: metothrexate-loaded HA (Met-HA), dexamethasone (Dex-M) and dexamethasone dispersed inside metothrexateloaded HA (Met-HA/Dex-M). Met-HA/Dex-M formulations could serve as an effective injectable drug, as it synergistically enhance local RA repair. Kim KS et al. ${ }^{41}$ assessed that intra-articular injection of tyramine modified hyaluronic acid encapsulating dexamethasone (DMT) resulted in successful treatment of RA with reduced pro-inflammatory cytokines such as interlukine-6, prostaglandin E2 and other types of cytokine levels in collagen-induced arthritis animal models. Yasuda ${ }^{42}$ hystologically compared the effects of HA in normal, osteoarthritis, and rheumatoid arthritis affected hip joints. In this study, explanted articular cartilage was incubated with heparin-binding fibronectin fragment (HBFN-f) producing higher levels of nitric oxide (NO) compared with normal cartilage. HA seems to block HBFN-f actions in OA and RA cartilages through interaction with some molecular factors such as CD44. HA, which targets CD44 highly expressed on OA and RA chondrocytes, could suppress catabolic actions by fibronectin fragments like HBFN-f in diseased cartilage. Saito et al. ${ }^{43}$ evaluated the patient-based database from a cross-sectional observational cohort in order to investigate if the intra-articular injection of HA is a potential clinical option for the treatment of RA. $14 \%$ of the patients in the database received corticosteroids or HA injections. The patients satisfaction rate of injection was 64 for corticosteroid injections and 59.3 for HA injections. HA and corticosteroid injections generally have similar efficacy rates; HA could be identified as a therapeutic option for RA.

Abate et al. ${ }^{44}$ investigated the role of $\mathrm{HA}$ injections in femoroacetabular impingement (FAI).

They reported the short-term results on hip pain and function after 2-ml intra-articular ultrasound-guided injection of hyaluronic acid at baseline and after 40 days in 23 hips affected by mild FAl. They evidenced significant pain reduction, anti-inflammatory medication consumption reduction and function improvement after HA injections.

\section{Conclusions}

There is lack of general consensus about the standardization of HA injections in hip OA osteoarthritis and other hip diseases.

We can state that: 
- The evidence suggest that this is the best conservative therapy for osteoarthritis before surgery indications, and it can act on pain relief without modifying the morphological structure of the pathological hip and natural history of the disease.

- There is no general consensus on number and timing of hip injections. Studies provide from one to four injections following a variable timing. Ultrasound guided injection is the safest approach.

- There is no relevant difference between low molecular weight and high molecular weight HA. The outcome after injection is comparable. No comparison has been made with medium molecular weight hyaluronic acid injections.

- If HA is associated to PRP o corticosteroids, pain relief is more rapid but it is not clear if this condition is persistent. There is no evidence on the efficacy of PRP and corticosteroid injections alone in hip osteoarthritis.

- The only improvement is on pain and walking distance. Radiographic changes are not present, and the progression towards surgical option is not modified.

- The efficacy on symptoms is demonstrated only in mild to moderate OA. Severe disease does not benefit from this treatment. However, studies on the early disease and on young populations have not been published yet.

- Concerning other hip joint conditions, only a few studies investigated the role of $\mathrm{HA}$ injections in rheumatoid arthritis or femoroacetabular impingement. No studies have been published On femoral head avascular necrosis or hip dysplasia.

- It is not clear whether a concomitant rheumatic disease such as rheumatoid arthritis or synovial inflammation could influence the clinical outcome of HA hip injections during osteoarthritis.

- HA in association with methotrexate or dexamethasone injections seems to be able to reduce synovial level of pro-inflammatory cytokines during rheumatoid arthritis.

- Published data do not allow us to consider HA able to modify the natural history of the hip disorders included in our review. We can only speculate about clinical improvement in terms of pain relief.

- Further studies need to be planned about HA injections and other hip congenital or acquired diseases, like femoral head avascular necrosis or hip dysplasia. To our knowledge, only a few case series reported conservative treatment of femoral osteonecrosis, but they investigated adipose-tissue derived stem cells. Considering synovial fluid cartilage metabolism in osteonecrosis of the femoral head, the impact of HA in hip disruption should be investigated.

All the procedures described in the present study comply with the Muscles, Ligaments and Tendons Journal. Basic principles and recommendations in clinical and field science research.

\section{References}

1. Sozzani S, Abbracchio MP, Annese V, et al. Chronic inflammatory diseases: do immunological patterns drive the choice of biotechnoloy drugs? A critical review. Autoimmunity. 2014; 47:287-306.

2. Feldmann M. Developmento of anti-TNF therapy for rheumatoid arthritis, Nat Rev Immunol. 2002,;2,364.

3. Choy EH, Panayi GS. N Engl J Med. 2001;344(12):907-16.

4. Beck M, Kalhor M, Leunig M, Ganz R. Hip morphology influences the pattern of damage to the acetabular cartilage: femoroacetabular impingement as a cause of early osteoarthritis of the hip. J Bone Joint Surg Br. 2005;87:10121018.

5. Ganz R, Parvizi J, Beck M, et al. Femoroacetabular impingement: a cause for osteoarthritis of the hip. Clin. Orthop Relat Res. 2007;465:46-52.

6. Meyer K, Palmer JW. On the nature of the ocular fluids. Amer Jour of Ophtalmol. 1936;19(10):859-865.

7. Endre A. Balazs. The physical properties of synovial fluid and the special role of Hyaluronic Acid. Disorders of the knee. 1974;63-75.

8. Balazs EA, Denlinger JL. Viscosupplementation: a new concept in the treatment of osteoarthritis. J Rheumatol. 1993; 39:3-9.

9. Laurent TC, Laurent UB, Fraser JR. The structure and function of hyaluronan: An overview. Immunol Cell Biol.1996;74(2):A17.

10. Fraser JR, Laurent TC, Laurent UB. Hyaluronan: its nature, distribution, functions and turnover. J Intern Med.1997;242 (1):27-33.

11. Henrotin $Y$, Raman $R$, Richette $P$, et al. Consensus statement on viscosupplementation with hyaluronic acid for the management of osteoarthritis. Semin Arthritis Rheum. 2015;45:140-9.

12. Hui AY, McCarty WJ, Masuda K, et al. A systems biology approach to synovial joint lubrication in health, injury, and disease. Wiley Interdiscip Rev Syst Biol Med. 2012;4:15-37.

13. Rhee DK, Marcelino J, Baker M, et al. The secreted glycoprotein lubricin protects cartilage surfaces and inhibits synovial cell overgrowth. J Clin Invest. 2005;115:622-31.

14. Ludwig TE, McAllister JR, Lun V, et al. Diminished cartilage-lubricating ability of human osteoarthritic synovial fluid deficient in proteoglycan 4: restoration through proteoglycan 4 supplementation. Arthritis Rheum. 2012;64:3963-71.

15. Saito S, Momohara S, et al. The intra-articular efficacy of hyaluronate injections in the treatment of rheumatoid arthritis. Mod Rheumatol. 2009.

16. Khan W, Khan M, Alradwan H, et al. Utility of Intra-articular Hip Injections for Femoroacetabular Impingement Orthop J Sports Med. 2015

17. Abate M, Scuccimarra T, Vanni D, et al. Femoroacetabular impingement: is hyaluronic acid effective? Knee Surg Sports Traumatol Arthrosc. 2014.

18. Kikuchi T, Yamada $\mathrm{H}$, et al. Effects of high molecular weight hyaluronan, on the distribution and movement of proteoglycan around chondrocytes cultured in alginate beads. Osteoarthritis Cartilage. 2001;9:351-356.

19. Moher D, Liberati A, Tetzlaff J, et al. Preferred reporting items for systematic reviews and meta-analyses: the PRISMA statement. Ann InternMed 2009.

20. Lùrati $A$, Laria $A$, Mazzocchi $D$, Re KA, Marrazza M, Scarpellini M. Effects of hyaluronic acid (HA) viscosupplementation on peripheral Th cells in knee and hip osteoarthritis. Osteoarthritis Cartilage. 2015;23(1):88-93.

21. Dallari D, Stagni C, Rani N, et al. Ultrasound-Guided Injection of Platelet-Rich Plasma and Hyaluronic Acid, Separately and in Combination, for Hip Osteoarthritis: A Randomized Controlled Study. Am J Sports Med. 2016;Mar;44(3):664-71. 
22. Battaglia M, Guaraldi F, Vannini F, Rossi G, Timoncini A, Buda R, Giannini S. Efficacy of ultrasound-guided intra-articular injections of platelet-rich plasma versus hyaluronic acid for hip osteoarthritis. Orthopedics. 2013;36(12):e1501-8.

23. Migliore A, Massafra U, Bizzi E, et al. Comparative, doubleblind, controlled study of intra-articular hyaluronic acid (Hyalubrix) injections versus local anesthetic in osteoarthritis of the hip. Arthritis Res Ther. 2009;11(6):R183.

24. Richette $\mathrm{P}$, Ravaud $\mathrm{P}$, Conrozier $\mathrm{T}$, et al. Effect of hyaluronic acid in symptomatic hip osteoarthritis: a multicenter, randomized, placebo-controlled trial. Arthritis Rheum. 2009;60(3): 824-30.

25. Qvistgaard E, Christensen R, Torp-Pedersen S, Bliddal H. Intra-articular treatment of hip osteoarthritis: a randomized trial of hyaluronic acid, corticosteroid, and isotonic saline. Osteoarthritis Cartilage. 2006;14(2):163-70.

26. Van den Bekerom MP, Rys B, Mulier M. Viscosupplementation in the hip: evaluation of hyaluronic acid formulations. Arch Orthop Trauma Surg. 2008;128(3):275-80.

27. Tikiz C, Unlü Z, Sener A, Efe M, Tüzün $C$. Comparison of the efficacy of lower and higher molecular weight viscosupplementation in the treatment of hip osteoarthritis. Clin Rheumatol. 2005;24(3):244-50.

28. Atchia I, Kane D, Reed MR, Isaacs JD, Birrell F. Efficacy of a single ultrasound-guided injection for the treatment of hip osteoarthritis. Ann Rheum Dis. 2011;70(1):110-6.

29. Migliore A, Martin LS, Alimonti A, Valente C, Tormenta S. Efficacy and safety of viscosupplementation by ultrasound-guided intra-articular injection in osteoarthritis of the hip. Osteoarthritis Cartilage. 2003;11(4):305-6.

30. Migliore A, Tormenta S, Martin Martin LS. The symptomatic effects of intra-articular administration of hylan G-F 20 on osteoarthritis of the hip: clinical data of 6 months follow-up. Clin Rheumatol. 2006;25(3):389-93.

31. Eyigör C, Pirim A, Eyigör S, Uyar M. Efficacy of intraarticular hyaluronic acid injection through a lateral approach under fluoroscopic control for advanced hip osteoarthritis. Agri. 2010;22(4):139-44.

32. Migliore A, Bizzi E, Massafra $U$, et al. Report from italian national register: reduction in nsaids consumption after intra-articular injections of hyaluronic products in patients with symptomatic hip osteoarthritis, Osteoarthritis and Cartilage, 2008.

33. Migliore A, Granata M, Tormenta S, et al. Hip viscosupplementation under ultra-sound guidance riduces NSAID consumption in symptomatic hip osteoarthritis patients in a long follow-up. Data from Italian registry. Eur Rev Med Pharmacol
Sci. 2011;15(1):25-34.

34. Conrozier T, Bertin P, Bailleul F. Clinical response to intra-articular injections of hylan G-F 20 in symptomatic hip osteoarthritis: the OMERACT-OARSI criteria applied to the results of a pilot study. Joint Bone Spine. 2006;73(6):705-9.

35. Conrozier T, Couris CM, Mathieu P, Correlation between patient self assessment of treatment efficacy (pate) and other outcome variables. Results of a standardized follow-up in patients treated with intra-articular injection of hyaluronic acid for hip osteoarthritis, Osteoarthritis and Cartilage, 2008.

36. Brocq O, Tran G, Breuil V, Grisot C, Flory P, Euller-Ziegler L. Hip osteoarthritis: short-term efficacy and safety of viscosupplementation by hylan G-F 20. An open-label study in 22 patients. Joint Bone Spine. 2002;69(4):388-91.

37. Vad VB, Sakalkale D, Sculco TP, Wickiewicz TL. Role of hylan G-F 20 in treatment of osteoarthritis of the hip joint. Arch Phys Med Rehabil. 2003;84(8):1224-6.

38. Gaston MS, Tiemessen CH, Philips JE. Intra-articular hip viscosupplementation with synthetic hyaluronic acid for osteoarthritis: efficacy, safety and relation to pre-injection radiographs. Arch Orthop Trauma Surg. 2007;127(10):899-903.

39. Migliore A, Bella A, Bisignani M, et al. Total hip replacement rate in a cohort of patients affected by symptomatic hip osteoarthritis following intra-articular sodium hyaluronate (MW 1,500-2,000 kDa) ORTOBRIX study. Clin Rheumatol. 2012;31(8):1187-96.

40. Reum Son A, Kim da Y, Hun Park S, et al. Direct chemotherapeutic dual drug delivery through intra-articular injection for synergistic enhancement of rheumatoid arthritis treatment. Sci Rep. 2015;1;5:14713.

41. Kim KS, Park SJ, Yang JA, Jeon JH, Bhang SH, Kim BS, Hahn SK. Injectable hyaluronic acid-tyramine hydrogels for the treatment of rheumatoid arthritis. Acta Biomater. 2011;7(2):666-74.

42. Yasuda T. Comparison of hyaluronan effects among normal, osteoarthritis, and rheumatoid arthritis cartilages stimulated with fibronectin fragment. Biomed Res. 2010;31(1):63-9.

43. Saito S, Momohara S, Taniguchi A, Yamanaka H. The intra-articular efficacy of hyaluronate injections in the treatment of rheumatoid arthritis. Mod Rheumatol. 2009;19(6):643-51.

44. Abate M, Scuccimarra T, Vanni D, Pantalone A, Salini V. Femoroacetabular impingement: is hyaluronic acid effective? Knee Surg Sports Traumatol Arthrosc. 2014;22(4):889-92

45. Padulo J, Oliva F, Frizziero A, Maffulli N. Muscles, Ligaments and Tendons Journal. Basic principles and recommendations in clinical and field Science Research: 2016 Update. MLTJ. 2016;6(1):1-5. 